Original

\title{
Fluoride availability and stability of Japanese dentifrices
}

\author{
Lina Naomi Hashizume ${ }^{\S}$, Ynara Bosco de Oliveira Lima ${ }^{\dagger}$, \\ Yoko Kawaguchi ${ }^{\ddagger}$ and Jaime Aparecido Cury ${ }^{\dagger}$

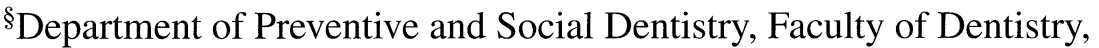 \\ Federal University of Rio Grande do Sul, Porto Alegre, RS, Brazil \\ ${ }^{\dagger}$ Laboratory of Oral Biochemistry, Faculty of Dentistry of Piracicaba, \\ State University of Campinas, Piracicaba, SP, Brazil \\ †Department of Oral Health Promotion, Graduate School, \\ Tokyo Medical and Dental University, Tokyo 113-8549
}

(Received 14 May and accepted 1 October 2003)

\begin{abstract}
The decline of dental caries in the industrialized countries can be attributed to widespread use of fluorides. The Japanese market share of dentifrices containing fluorides has increased from $12 \%$ in 1985 to $77 \%$ in 2000 . For a fluoride dentifrice to be effective in the control of dental caries, an adequate concentration of the fluoride must be soluble. Several Japanese fluoride dentifrices have in their formulations calcium phosphate as an abrasive, which may react with fluoride. This study was designed to evaluate the availability and stability of fluoride in the most consumed dentifrices in Japan. The analyses were made when the dentifrices were purchased (fresh samples) and after one year of storage (aged samples) at room temperature $\left(21.8 \pm 3.6^{\circ} \mathrm{C}\right)$. Total fluoride and soluble fluoride was determined using an ion specific electrode. All dentifrices showed similar concentrations of total fluoride in fresh and aged samples in accordance with the Japanese Legislation (content of less than 1,000 ppm F). Some dentifrices, with dicalcium phosphate as abrasive, showed decreasing concentrations of total soluble fluoride and increasing amounts of insoluble fluoride over time. Although most of the Japanese fluoridated dentifrices evaluated in this study contain unstable fluoride, they were found to have
\end{abstract}

Correspondence to Dr. Lina Naomi Hashizume, Department of Preventive and Social Dentistry, Faculty of Dentistry, Federal University of Rio Grande do Sul, Rua Ramiro Barcelos, 2492 Porto Alegre, RS, Brazil 90035-003

Tel: +55-51-3316-5193

Fax: +55-51-3316-5002

E-mail: lhashizume@yahoo.com sufficient concentrations of soluble fluoride to be effective in preventing dental caries. ( $\mathrm{J}$ Oral Sci. 45, 193199, 2003)

Key words: dentifrice; fluoride; caries prevention; stability.

\section{Introduction}

The prevalence of dental caries in developed countries has declined over the past several decades (1). Several studies are in agreement that the main reason for the reduction of caries is the greater availability of fluoride in the oral environment, particularly the increasing use of fluoridated dentifrice over the last 25 years $(2,3)$.

In most Western industrialized countries, the percentage of fluoridated dentifrices of all dentifrice sales is above $90 \%$, with an upward trend (4). However, in order for the dentifrice to be efficient, it is important that the fluoride is soluble in the formulation and it is regularly present in the oral cavity, so it can interfere with the phenomena of enamel-dentine demineralization and remineralization (5).

The Japanese market share of dentifrices containing fluorides, such as sodium monofluorphosphate (MFP) or sodium fluoride, has increased from $12 \%$ in 1985 to $77 \%$ in 2000 (6). Until 1994, the market share of fluoridated toothpastes was only $46 \%$, but after the adding of fluoride into the two most popular Japanese toothpastes (White \& White $^{\circledR}$ and Dentor ${ }^{\circledR}$; Lion Company), a sudden increase in this share was verified. Several Japanese fluoride dentifrices have in their formulations dicalcium phosphate as an abrasive, which may react with fluoride ions released 
from MFP.

The Japanese Legislation establishes the maximum fluoride content of $1000 \mathrm{ppm} F$ that is permitted in a dentifrice (7). However, the concentration of fluoride contained in the dentifrice is not indicated on the package.

For a fluoridated dentifrice to be effective in controlling dental caries, an adequate concentration of fluoride must be soluble (as fluoride ion or MFP). The soluble forms of fluoride are able to interfere with the dynamics of the caries process, reducing the demineralization and activating the remineralization of dentine and enamel. Some forms of fluoride may link to the abrasive contained in the dentifrice formulation. Thus, the total concentration of fluoride is not the concentration of the soluble and active fluoride contained in the toothpaste.

Rules and standards concerning the activity and stability of fluoride in toothpastes have been published. The US Food and Drug Administration requires that the soluble fluoride ion $\left(\mathrm{F}^{-}\right)$for $\mathrm{NaF}$ toothpastes and the soluble $\mathrm{F}$ ions $\left(\mathrm{F}^{-}\right.$plus $\mathrm{PO}_{3} \mathrm{~F}^{2-}$ ) for MFP toothpastes shall be not less than $60 \%$ of the total fluoride content. The Standards Association of Australia and the European Community have produced rules for the minimum amount of soluble fluoride that must be present in fluoride toothpaste throughout its shelf life. These rules require that at least $60 \%$ of the total fluoride content be present as a soluble ion either as $\mathrm{F}^{-}$or $\mathrm{PO}_{3} \mathrm{~F}^{2-}$. The latter ion is rapidly hydrolyzed in the mouth to provide $F^{-}$. However, some hydrolysis occurs slowly from the time of manufacture and combines with abrasives or other toothpaste constituents to form insoluble compounds so that the total soluble fluoride concentration falls with time (8). Futhermore, with age there is a considerable loss in the soluble fluoride concentration found in dentifrice (9).

The minimum requirement for the anti-caries effect of a dentifrice is based on the available and stable fluoride in the formulation. However, the Japanese legislation only establishes the maximum fluoride content $(0.10 \%)$, without specifying quality. No studies about the availability and stability of fluoride in Japanese dentifrices were found in the literature, justifying this study.

The aim of the present study was to evaluate the availability and stability of fluoride in all the forms (i.e. total fluoride, total soluble fluoride, ion fluoride, MFP and insoluble fluoride) in the most consumed dentifrices in Japan.

\section{Materials and Methods}

\section{Preparation of the samples}

Five dentifrices, the most consumed on the Japanese market, were evaluated (Table 1). Three tubes of each brand were purchased from different stores from the Tokyo area. Their packages indicated the abrasive used, salt of fluoride present but not its concentration. Information about fluoride concentration was obtained from the Japan Dentifrice Manufacturers'Association (10). The samples were codified with letters A, B, C, D and E allowing a blind

Table 1 Information on each dentifrice analyzed in this study

\begin{tabular}{|c|c|c|c|c|c|}
\hline $\begin{array}{l}\text { Commercial brand } \\
\text { (manufacturer) }\end{array}$ & Code & $\begin{array}{c}\text { Fluoridated } \\
\text { agent }\end{array}$ & $\begin{array}{c}\text { Fluoride content } \\
\text { expected** }\end{array}$ & $\begin{array}{l}\text { Abrasive } \\
\text { agent }\end{array}$ & $\begin{array}{c}\text { Lot } \\
\text { Number }\end{array}$ \\
\hline $\begin{array}{l}\text { White and White } \\
\text { (Lion) }\end{array}$ & A & MFP* & $960 \mu \mathrm{g} \mathrm{F} / \mathrm{g}$ & $\begin{array}{l}\text { Dibasic calcium phosphate } \\
\text { dihydrate and alumina }\end{array}$ & $\begin{array}{l}\text { W1287 } \\
\text { W1307 } \\
\text { W1264 }\end{array}$ \\
\hline $\begin{array}{l}\text { Gum Dental Paste } \\
\text { (Sun Star) }\end{array}$ & B & MFP & $924 \mu \mathrm{g} \mathrm{F} / \mathrm{g}$ & $\begin{array}{c}\text { Dibasic calcium phosphate } \\
\text { dihydrate }\end{array}$ & $\begin{array}{l}627 \mathrm{~J} \\
212 \mathrm{~L} \\
726 \mathrm{~N}\end{array}$ \\
\hline $\begin{array}{l}\text { PC Clinica } \\
\text { (Lion) }\end{array}$ & $\mathrm{C}$ & MFP & $960 \mu \mathrm{g} \mathrm{F} / \mathrm{g}$ & Alumina & $\begin{array}{l}\text { A1197 } \\
\text { A1165 } \\
\text { A1181 }\end{array}$ \\
\hline $\begin{array}{l}\text { Clear Clean } \\
\text { (Kao) }\end{array}$ & $\mathrm{D}$ & MFP & $883 \mu \mathrm{g} \mathrm{F} / \mathrm{g}$ & Silica & $\begin{array}{l}61621 \\
61611 \\
31871 \\
\end{array}$ \\
\hline $\begin{array}{l}\text { Dentor } \\
\text { (Lion) }\end{array}$ & $\mathrm{E}$ & MFP & $960 \mu \mathrm{g} \mathrm{F} / \mathrm{g}$ & $\begin{array}{l}\text { Dibasic calcium phosphate } \\
\text { dihydrate }\end{array}$ & $\begin{array}{l}\text { A1126 } \\
\text { A1115 } \\
\text { W1088 }\end{array}$ \\
\hline
\end{tabular}

* MFP = monofluorphosphate

** The fluoride content was not specified on the package, these data were obtained from the Report of the Japan Dentifrice Manufacturers' Association. 
analysis. The initial analyses were carried out at the time of acquisition (fresh samples). Then, the tubes of the dentifrices were packed into a box and stored on a shelf of our laboratory for one year. The room temperature was measured every day for this period and the average (Mean $\pm \mathrm{SD}$ ) obtained was $21.8 \pm 3.6^{\circ} \mathrm{C}$. After this storage period (aged samples), final analyses were done. This study was conducted from October 2001 to October 2002.

\section{Determination of Total Fluoride}

The total fluoride (TF) represents the sum of the total soluble fluoride concentrations plus the insoluble fluoride concentrations. Insoluble fluoride represents the fluoride inactivated by the abrasive of the dentifrice. TF was determined with an ion specific electrode after diffusion with hexamethyldisiloxane (HMDS), because most of the dentifrices analyzed contained the abrasive calcium and aluminum that interferes with the direct analysis (11). The method described by Taves was used (12). A shortened plastic cap of $17 \times 100 \mathrm{~mm}$ (Falcon 2030; Becton, Dickinson and Co, Franklin Lakes, USA) was fixed with petroleum jelly (Vaseline) in the center of a petri dish of $60 \times 15$ mm (Falcon 1007; Becton, Dickinson and Co, Franklin Lakes, USA). The dentifrice was weighed ( \pm $0.01 \mathrm{mg}$ ) directly in the bottom of the petri dish and $3 \mathrm{ml}$ of distilled and deionized water (DDW) was added to it. A volume of $0.1 \mathrm{ml}$ of $1.65 \mathrm{~N} \mathrm{NaOH}$ was placed in the cap. The petri dish was closed, and the cap was sealed with Vaseline. A volume of $1.0 \mathrm{ml}$ of $\mathrm{H}_{2} \mathrm{SO}_{4}$ saturated with HMDS was added to the mixture (dentifrice- $\mathrm{H}_{2} \mathrm{O}$ ) through a hole made in the cap. The hole was quickly sealed with Vaseline and covered with a small piece of parafilm. HMDS enhances the rate of diffusion (separation) of fluoride by the formation of trimethylfluorosilane (TMFS), which is highly volatile and hydrophobic and thus escapes from the acidified sample. The diffusion process was carried out for $12 \mathrm{~h}$ (overnight) at room temperature with $80 \mathrm{rpm}$ rotatory motion. The volatile TMFS is trapped in the $\mathrm{NaOH}$ and released fluoride in the solution. This solution was dried at $60^{\circ} \mathrm{C}$ for $2 \mathrm{~h}$, by placing the cap in an oven. A volume of $0.4 \mathrm{ml}$ of $0.66 \mathrm{~N}$ acetic acid was added to a plastic tube $17 \times 100 \mathrm{~mm}$ (Falcon 2017; Becton, Dickinson and Co, Franklin Lakes, USA), which was closed with the cap that contained the dry sample. The tube was inverted, vortexed, opened and placed in contact with the fluoride ion specific electrode for analysis. The analyses were done in triplicate and TF represent the concentration of total soluble fluoride (TSF) plus insoluble fluoride (IS). IS represents fluoride inactivated by the dentifrice abrasive. The calibration standards were also acid-diffused.

\section{Determination of soluble fluoride}

The concentrations of TSF and as fluoride ion $\left(\mathrm{F}^{-}\right)$were determined directly with an ion specific electrode with or without prior acid hydrolysis, respectively, according to the modified procedure described by Cury et al. (13). One hundred $\mathrm{mg}$ of each dentifrice was weighed $( \pm 0.01 \mathrm{mg})$ in graduated centrifuge plastic tubes. Ten $\mathrm{ml}$ of DDW was added to each tube that was then vigorously shaken for 20 seconds to obtain a homogeneous suspension. The suspension was centrifuged at $3000 \times g$ for 10 minutes, the precipitated discarded and the supernatant kept for the analyses.

To determine the concentration of TSF, $0.06 \mathrm{ml}$ of the supernatant was transferred to a plastic test tube and 0.190 $\mathrm{ml}$ of DDW was added to it. A volume of $0.25 \mathrm{ml}$ of $2 \mathrm{M}$ $\mathrm{HCl}$ was added to the tube that was heated at $45^{\circ} \mathrm{C}$ for 1 $\mathrm{h}$ for hydrolysis of fluoride bound to MFP moiety. Next, $0.5 \mathrm{ml}$ of $1 \mathrm{M} \mathrm{NaOH}$ and $1.0 \mathrm{ml}$ of TISAB II $(0.75 \mathrm{M}$ acetate buffer, $\mathrm{pH} 5.0$, containing $1 \mathrm{M} \mathrm{NaCl}$ and $0.4 \%$ of CDTA) were added to the tube. The determination of fluoride ions originally present in the dentifrice and that released from MFP by the acid hydrolysis was made in this tube. This solution was placed in contact with the fluoride ion specific electrode for the analysis.

To determine the concentration of $\mathrm{F}^{-}, 0.06 \mathrm{ml}$ of the supernatant was transferred to another plastic test tube and $0.190 \mathrm{ml}$ of DDW, $1.0 \mathrm{ml}$ of TISAB II, $0.5 \mathrm{ml}$ of $1 \mathrm{M} \mathrm{NaOH}$ and $0.25 \mathrm{ml}$ of $2 \mathrm{M} \mathrm{HCl}$ were added in this sequence. The concentration of $\mathrm{F}^{-}$was determined immediately using the electrode.

Thus, mathematically TSF $=\mathrm{F}^{-}+\mathrm{MFP}$ and MFP $=\mathrm{TSF}$ - $\mathrm{F}^{-}$. Considering that $\mathrm{TF}=\mathrm{TSF}+\mathrm{IS}$, it is possible to calculate the percentage of fluoride inactivated (IS) by the dentifrice abrasive.

\section{Fluoride analysis}

A specific electrode for fluoride ORION 96-09 connected to an ion analyzer ORION EA 940 (Orion Research Inc., Boston, USA) was used for all the analyses. Previously, calibration was done in triplicate with fluoride standard solutions, containing $0.8,1.6,3.2$ and $6.4 \mu \mathrm{g} \mathrm{F} / \mathrm{ml}$ for total fluoride determination and $0.25,0.5,1.0,2.0$ and 4.0 $\mu \mathrm{g} \mathrm{F} / \mathrm{ml}$ for the determination of the soluble forms of fluoride present in the dentifrices. The analyses were validated using internal standards and a coefficient variation lower than $3 \%$ was considered as acceptable.

\section{Statistical analysis}

The calibration and concentrations determined were tested in the linear regression curve, using the software EXCEL (Microsoft), where a calculation program 
transformed the values of $\mathrm{mV}$ (millivolts) provided by the electrode in $\mu \mathrm{g} \mathrm{F/g}$ for each dentifrice. The means and standard deviations were calculated with EXCEL.

\section{Results}

Tables 2 and 3 show the results (average of $n=3$ ) of the concentrations of total fluoride and the total soluble fluoride as MFP and ion fluoride, in the fresh and aged samples, respectively. With regard to the concentrations of total fluoride, there was no significant difference between the fresh and the aged samples. The fresh samples and the samples used after a year of storage (aged) at room temperature $(21.78 \pm 3.56)$ had similar concentrations of total fluoride contained in the dentifrices. Both results are in accordance with the Japanese Legislation.

The concentrations of total soluble fluoride are shown in Fig. 1. The aged samples of dentifrices A, B, C and E showed a decrease of total soluble fluoride concentration as a result of storage time in ambient temperature. Only dentifrice $\mathrm{D}$ showed similar values for total soluble fluoride concentration in the fresh and aged samples.

With regard to concentration of fluoride as MFP, dentifrices B, C, D and E had reductions of $20.2 \%, 4.8 \%$, $3.9 \%$ and $38.7 \%$, respectively. Only dentifrice A had an increase $(2.8 \%)$ in the concentration of MFP. There were decreases of the concentration of free fluoride $\left(\mathrm{F}^{-}\right)$in aged samples of dentifrices A (87.5\%), B (15.6\%) and C (1.7\%), while dentifrices D and $\mathrm{E}$ showed increases of $31.6 \%$ and

Table 2 Concentration $(\mu \mathrm{g}$ F/g) of total and soluble fluoride found in fresh samples of the dentifrices $($ Mean \pm SD; $\mathrm{n}=3$ )

\begin{tabular}{|c|c|c|c|}
\hline \multirow{2}{*}{ DENTIFRICES } & \multirow{2}{*}{$\begin{array}{c}\text { TOTAL } \\
\text { FLUORIDE }\end{array}$} & \multicolumn{2}{|c|}{ SOLUBLE FLUORIDE } \\
\cline { 3 - 4 } & & FI & MFP \\
\hline $\mathbf{A}$ & $1008.5 \pm 46.3$ & $190.7 \pm 17.5$ & $688.8 \pm 66.2$ \\
\hline $\mathbf{B}$ & $993.5 \pm 38.8$ & $188.9 \pm 10.0$ & $665.2 \pm 11.3$ \\
\hline $\mathbf{C}$ & $985.5 \pm 25.6$ & $82.9 \pm 8.4$ & $730.0 \pm 22.5$ \\
\hline $\mathbf{D}$ & $942.0 \pm 34.8$ & $135.5 \pm 8.0$ & $783.2 \pm 32.1$ \\
\hline $\mathbf{E}$ & $998.3 \pm 29.6$ & $154.3 \pm 19.4$ & $781.3 \pm 49.9$ \\
\hline
\end{tabular}

$\mathrm{FI}=$ fluoride ion

MFP = fluoride as monofluorphosphate

Table 3 Concentration ( $\mu \mathrm{g} \mathrm{F/g})$ of total and soluble fluoride found in aged samples of the dentifrices $($ Mean \pm SD; $\mathrm{n}=3$ )

\begin{tabular}{|c|c|c|c|}
\hline \multirow{2}{*}{ DENTIFRICES } & \multirow{2}{*}{$\begin{array}{c}\text { TOTAL } \\
\text { FLUORIDE }\end{array}$} & \multicolumn{2}{|c|}{ SOLUBLE FLUORIDE } \\
\cline { 3 - 4 } & & FI & MFP \\
\hline $\mathbf{A}$ & $995.3 \pm 144.6$ & $23.9 \pm 1.5$ & $708.6 \pm 35.5$ \\
\hline $\mathbf{B}$ & $851.7 \pm 40.6$ & $159.4 \pm 11.6$ & $553.6 \pm 26.4$ \\
\hline $\mathbf{C}$ & $969.1 \pm 34.7$ & $119.2 \pm 9.5$ & $696.4 \pm 66.4$ \\
\hline $\mathbf{D}$ & $975.4 \pm 133.7$ & $177.0 \pm 14.4$ & $753.6 \pm 33.4$ \\
\hline $\mathbf{E}$ & $1059.0 \pm 18.9$ & $234.1 \pm 43.7$ & $563.5 \pm 79.6$ \\
\hline
\end{tabular}

FI = fluoride ion

MFP = fluoride as monofluorphosphate 
$51.7 \%$, respectively, in the free fluoride concentration of aged samples.

Fig. 2 presents the percentage of insoluble fluoride contained in the fresh and aged samples of each dentifrice. In the fresh samples, the percentages of insoluble fluoride were low, varying from $2.6 \%$ to $14.0 \%$. All the aged samples of dentifrices had increases in these percentages after one year of storage. Compared with their initial percentages of insoluble fluoride, dentifrices A, B, C, D and $\mathrm{E}$ had increases of $106.3 \%, 16.1 \%, 16.4 \%, 78.0 \%$ and $294.8 \%$, respectively. Although dentifrice D had a $78.0 \%$ increase, the percentage of insoluble fluoride after oneyear remained low $(4.6 \%)$.

All dentifrices showed a tendency towards decreases in the concentration of total soluble fluoride and an increase in the percentage of insoluble fluoride after one year of storage at room temperature.

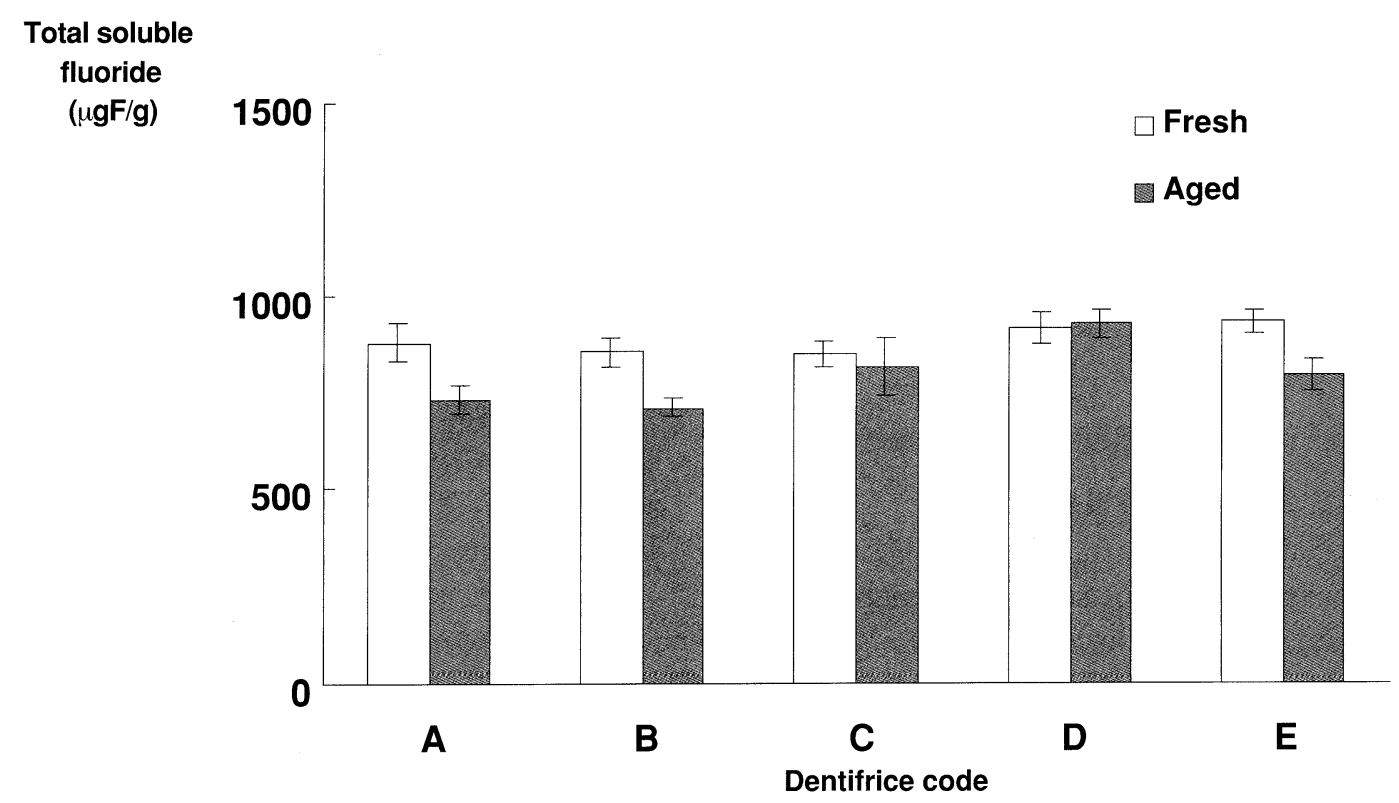

Fig. 1 Concentration $(\mu \mathrm{g} \mathrm{F/g})$ of total soluble fluoride found in fresh and aged samples of the dentifrices evaluated (Bars indicate $\mathrm{SD} ; \mathrm{n}=3)$.

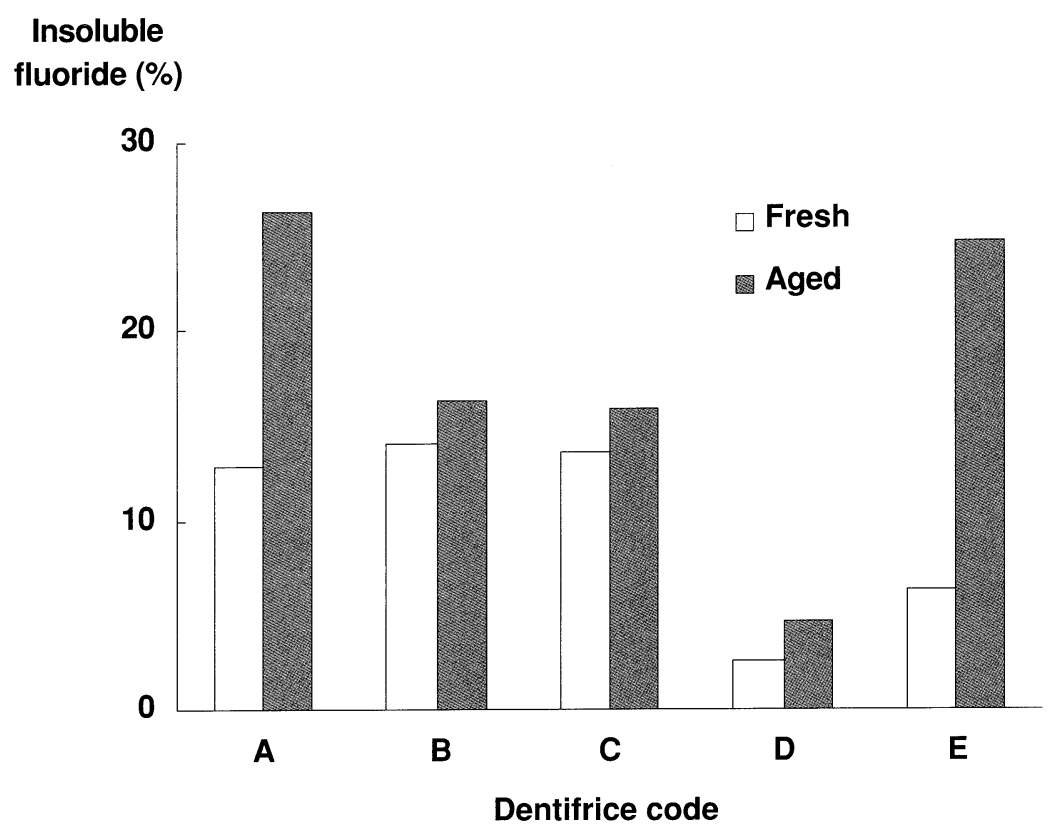

Fig. 2 Percentages of insoluble fluoride found in fresh and aged samples of the dentifrices evaluated. 


\section{Discussion}

The decline of dental caries in industrialized countries can be attributed to the widespread use of fluorides, mainly in the form of fluoride dentifrices (4). Approximately 500 million people of the world's population now use fluoride dentifrice making it by far the most important fluoride delivery system (14). Tooth brushing with fluoride dentifrice has become an important public health measure in preventing caries (15).

The regular application of fluoride from toothpaste confers protection primarily by exerting a topical effect on the erupted teeth. Fluoride interacts with the plaque/tooth system in four ways: reduction of mineral solubility, inhibition of mineral dissolution, inhibition of acid production by plaque bacteria and the promotion of remineralization $(16,17)$. However, the total fluoride contained in the dentifrice is not completely available. For the dentifrice to be effective in the prevention of caries, not only quantity but also quality of fluoride is important.

The majority of dentifrices contain either fluoride in their composition as the fluoride ion $(\mathrm{NaF})$ or as MFP, which are considered active forms for controlling caries (8). Fluoride in dentifrices must be in the dentifrice in a soluble form to guarantee activity against caries (18). Depending on the formulation, part of the fluoride may be inactive and this occurs mainly in the presence of calcium $\left(\mathrm{Ca}^{++}\right)$ and fluoride ions $\left(\mathrm{F}^{-}\right)$. Although MFP is more stable in the presence of $\mathrm{Ca}^{++}$, because fluoride is linked covalently to phosphate, it undergoes hydrolysis over time and releases $\mathrm{F}^{-}$, which reacts with $\mathrm{Ca}^{++}$to form insoluble fluoride that is inactive against caries (19).

Analyzing the five most sold Japanese fluoridated dentifrices, we verified that the total fluoride concentrations in fresh samples on the individual dentifrices were reasonably close to the values provided by the Japan Dentifrice Manufacturers' Association (10). There was no significant variation in concentration as a result of a storage time of one year. This non-variation of the total was expected, but there may be chemical changes from one form of fluoride to another as a result of product stability.

The concentration of total soluble fluoride in dentifrices A, B and E showed reductions as a result of time (Fig. 1). However, dentifrice $\mathrm{C}$ and $\mathrm{D}$ did not undergo alterations after one year. This should be attributed to the fact that these dentifrices have alumina (dentifrice $\mathrm{C}$ ) and silica (dentifrice D) as abrasive in their formulations. Alumina and silica allow the fluoride concentration in a dentifrice to remain stable, independent of whether it is in the F-form or in MFP. On the other hand, the decrease of TSF in dentifrices A, B and E should be attributed to the fact that these dentifrices are composed of MFP, which undergoes hydrolysis over time producing $\mathrm{F}^{-}$which turn is inactivated by the $\mathrm{Ca}^{++}$of the dicalcium phosphate used as an abrasive (9,19-21). Although the data of this study have shown decreases of TSF in dentifrices A, B and E over the storage time, the minimum concentration attained has contributed to the decline of dental caries (22).

Dentifrices A, B and C had percentages of insoluble fluoride that were greater than $10 \%$, while for dentifrices $\mathrm{D}$ and $\mathrm{E}$ the percentages were lower than $10 \%$ (Fig. 2). After one year of storage, dentifrices $\mathrm{A}$ and $\mathrm{E}$ increased their percentages to $106.3 \%$ and $294.8 \%$, respectively. This may be explained by the hydrolysis of MFP that releases $\mathrm{F}^{-}$, which links with the calcium of the abrasive resulting in insoluble fluoride $(9,19,23)$.

Stability was evaluated in this study over a period of one year, however it should be emphasized that the estimated time that dentifrices stay on the shelves of the stores is less than this evaluated period. This shelf time of the dentifrices would be sufficient for no loss of soluble fluoride and indicates that dentifrices can be effective in preventing dental caries.

Over previous decades, the Japanese government and dental professionals did not encourage the population to use fluoride products and their usages were strictly limited. The high caries rate of Japanese children in the 1970's and 1980's, compared to Western industrialized countries, may be attributed to the lack in the fluoride products usage $(24,25)$. However, the situation related to fluoride dentifrices in Japan has been changed since the beginning of the 1990's. In 1993, the market share for fluoride dentifrices was only $43 \%$; however, this percentage reached $77 \%$ in 1999 with an upward tendency (6).

The acceptance of fluoridated dentifrices is changing in Japan, and the effect of these changes can be observed in the increase of fluoridated dentifrices in the market share and the sudden increase in people consuming these products. However, the five most consumed dentifrices evaluated in this study did not specify the fluoride content on their packages. For the Japanese consumers to make informed choices before purchase of a fluoride dentifrice, the packages should be clear in showing the fluoride concentration contained in the product.

The results of this study suggest that the five most consumed Japanese fluoridated dentifrices contain a sufficient concentration of soluble fluoride, and may be used to prevent and control dental caries.

\section{Acknowledgments}

We would like to thank Mr. Waldomiro Vieira Filho, technician of the Laboratory of Oral Biochemistry, Faculty of Dentistry of Piracicaba, State University of Campinas 
for help with the initial analyses.

\section{References}

1. Birkeland JM, Haugejorden O, von der Fehr, FR (2000) Some factors associated with the caries decline among Norwegian children and adolescents: age-specific and cohort analyses. Caries Res 34, 109-116

2. Rolla G, Ogaard B, Cruz RA (1991) Clinical effect and mechanism of cariostatic action of fluoridecontaining toothpastes: a review. Int Dent J 41, 171174

3. Clarkson JJ (2000) International collaborative research on fluoride. J Dent Res 79, 893-904

4. World Health Organization (1994) Fluorides and oral health: Report of a WHO Expert Committee on oral health status and fluoride use. WHO Technical Report Series 846, Geneva, 1-37

5. ten Cate JM, Featherstone JDB (1996) Physicochemical aspects of fluoride-enamel interactions. In Fluoride in Dentistry, 2nd ed, Fejerskov O, Ekstrand J, Burt BA eds, Munksgaard, Copenhagen, 252-269

6. Lion Company (2000) Information for life. Oral Health Journal 21 (referred from internet: http://www.lion.co.jp/oral/ohj/ohj0021b.htm)

7. Kimura T, Morita M, Kinoshita T, Tsuneishi M, Akagi T, Yamashita F, Watanabe T (2001) Fluoride intake from food and drink in Japanese children aged 1-6 years. Caries Res 35, 47-49

8. Richards A, Banting DW (1996) Fluoride toothpastes. In Fluoride in Dentistry, 2nd ed, Fejerskov O, Ekstrand J, Burt BA eds, Munksgaard, Copenhagen, 352-372

9. de Freitas JF (1984) Fluoride stability in toothpastes. Aust Dent J 29, 30-35

10. Japan Dentifrice Manufacturers' Association (1999) Report of Japan Dentifrice Manufacturers' Association, Tokyo, 1-14 (in Japanese)

11. Hattab FN (1989) Analytical methods for the determination of various forms of fluoride in toothpastes. J Dent 17, 77-83

12. Taves DR (1968) Separation of fluoride by rapid diffusion using hexamethyldisiloxane. Talanta 15, 969-974

13. Cury JA, Guimarães LOC, Arbex ST, Moreira BW (1981) Analysis of fluoride dentifrices: composition and chemical forms of fluorides found in Brazilian products. Revista da APCD 35, 142-147 (in Portuguese)

14. Davies RM, Holloway PJ, Ellwood RP (1995) The role of fluoride dentifrices in a national strategy for the oral health of children. Br Dent J 5, 84-87

15. König KG (1993) Role of fluoride toothpastes in a caries-preventive strategy. Caries Res 27, 23-28

16. Dawes C, ten Cate JM (1990) International symposium on fluorides: mechanisms of action and recommendation for use. J Dent Res 69 Special Issue, 505-836

17. Shellis RP, Duckworth RM (1994) Studies on the cariostatic mechanisms of fluoride. Int Dent J 44, 263-273

18. ADA - Council on Dental Therapeutics (1985) Guidelines for the acceptance of fluoride-containing dentifrices. J Am Dent Assoc 110, 545-547

19. Cury JA (1986) Fluoride stability in the Brazilian dentifrices. Revista Gaúcha de Odontologia 34, 430-432 (in Portuguese)

20. Orth R.M, Assaf AV, Zanin L, Mialhe FL, Klein ALL, Medina MRJ, Pardi V (2001) Concentration of fluoride in the main marketed toothpastes in Brazil and the impact of the new decree of regulation. Revista Odonto Ciência 16, 27-33 (in Portuguese)

21. Conde NCO, Rebelo MA, Cury JA (2003) Evaluation of the fluoride stability of dentifrices sold in Manaus, AM, Brazil. Pesqui Odontol Bras (in press)

22. Margolis HC, Moreno EC, Murphy BJ (1986) Effect of low levels of fluoride in solution on enamel demineralization in vitro. J Dent Res 65, 23-29

23. Duarte FF, Pisaneschi E, Cury JA (1999) Evaluation of fluoride contained in the most consumed dentifrices in Brazil and commercialized in the five regions of the country. Revista da ABOPREV 2, 310 (in Portuguese)

24. Tsurumoto A, Wright FAC, Kitamura T, Fukushima M, Campain AC, Morgan MV (1998) Cross-cultural comparison of attitudes and opinions on fluorides and fluoridation between Australia and Japan. Community Dent Oral Epidemiol 26, 182-193

25. Federation Dentaire International (1985) Changing patterns of oral health and implications for oral health manpower: Part 1. Technical report 24. Int Dent J 35, 234-251 